\title{
AN ANALYTICAL DELAY MODEL FOR RLC INTERCONNECTS
}

\author{
Andrew B. Kahng* and Sudhakar Muddu* \\ UCLA Computer Science Department, Los Angeles, CA 90095-1596 USA \\ abk@cs.ucla.edu,sudhakar@cs.ucla.edu
}

\begin{abstract}
We develop an analytical delay model based on first and second moments to incorporate inductance effects into the delay estimate for interconnection lines. Delay estimates using our analytical model are within $15 \%$ of SPICE-computed delay across a wide range of interconnect parameter values. We also extend our delay model for estimation of source-sink delays in arbitrary interconnect trees. For the small tree topology considered, we observe improvements of at least $18 \%$ in the accuracy of delay estimates when compared to the Elmore model (which is independent of inductance), even though our estimates are as easy to compute as Elmore delay. The speedup of delay estimation via our analytical model is several orders of magnitude when compared to a simulation methodology such as SPICE.
\end{abstract}

\section{INTRODUCTION}

Accurate calculation of propagation delay in VLSI interconnects is critical to the design of high speed systems. Current techniques are based on either simulation or (closed-form) analytical formulas. Simulation tools such as SPICE give the most accurate insight into arbitrary interconnect structures, but are computationally expensive. Transient simulation of lossy interconnects based on convolution techniques is presented in $[8,12]$. Faster techniques based on moment computations are proposed in $[11,16,17]$. Since these methods are too expensive to be used during iterative layout optimization, the Elmore delay [2] approximation (which represents the first moment of the transfer function) is now widely used in the performancedriven design of clock distribution and Steiner global routing topologies. However, Elmore delay cannot accurately estimate the delay for $R L C$ interconnect lines, i.e., the representation for interconnects whose inductive impedance cannot be neglected $[4,6]$. Typically, the Elmore delay formula gives good estimates if interconnect lines are $R C$ or overdamped, but gives overestimates for $R L C$ or underdamped interconnects. This inaccuracy can be harmful for current performance-driven routing methods which try to optimize interconnect segment lengths and widths (as well as drivers and buffers) based on estimated delays.

This paper gives a new and accurate analytical delay estimate for distributed $R L C$ interconnects which considers the effect of inductance. Previous moment-based analysis of $R L C$ lines (e.g., $[9,8]$ ) can derive a delay estimate only after simulating the response, rather than from an analytical formula. To validate our analysis and delay formula, we model VLSI interconnect lines having various combinations of source and load parameters, and obtain delay estimates from SPICE, Elmore delay and the proposed analytical delay model. The delay estimate using SPICE is extracted from a computed response at the specified node, whereas the other two models are closedform expressions. Over our range of test cases, Elmore delay estimates can be quite far from the SPICE-computed delays, while our analytical delay model estimates are within $15 \%$ of SPICE delays. We also extend our delay model to estimate source-sink delays in arbitrary interconnect trees. For the small tree topology considered, our delay estimates are again within $18 \%$ of SPICE-computed delays, while Elmore delay estimates

\footnotetext{
*Partially supported by NSF MIP-9257982.
}

vary by as much as $35 \%$ from SPICE-computed delays. Since our analytical model has the same time complexity as the Elmore model, we believe that it can be useful in present-day performance-driven routing methodologies.

\section{PREVIOUS ANALYTICAL DELAY MODELS}

The transfer function of an $R L C$ interconnect line with source and load impedance (Figure 1) can be obtained using the ABCD parameters [1] as

$$
H(s)=\frac{1}{\left(1+\frac{Z_{S}}{Z_{T}}\right) \cosh (\theta h)+\left(\frac{Z_{S}}{Z_{0}}+\frac{Z_{n}}{Z_{T}}\right) \sinh (\theta h)}
$$

where $\theta=\sqrt{(r+s l) s c}$ is the propagation constant and $Z_{0}=$ $\sqrt{\frac{R+s L}{s C}}$ is the characteristic impedance; $r=\frac{R}{h}, l=\frac{L}{h}, c=\frac{C}{h}$ are resistance, inductance, and capacitance per unit length and $h$ is the length of the line. To compute the $R L C$ line response from the transfer function, the method of Padé approximation has been used by, e.g., $[9,10]$. The output transfer function is expanded into a Maclaurin series of $s$ around $s=0$, and the series is truncated to desired order. In general, analytical computation of the exact voltage response is very tedious and is usually in the form of an infinite series.

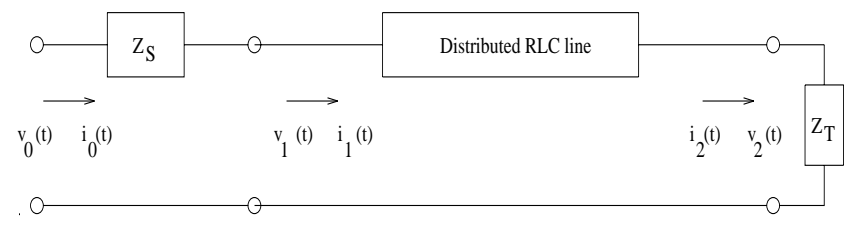

Figure 1. 2-port model of a distributed $R L C$ line with source impedance $Z_{S}$ and load impedance $Z_{T}$.

Efficient delay estimates for $R C$ lines are typically derived by considering a single interconnect line with resistive source and capacitive load impedances; delay formulas for an interconnect tree entail recursive application of the formula for a single line. The analytical Elmore delay [2] estimate, Sakurai's heuristic delay formula $[14,15]$ and the single pole delay estimates of [3] have been widely used.

- Elmore delay is defined to be the first moment of the system impulse response, i.e., the coefficient of $s$ or the first moment in the system transfer function $H(s)$. Applying this definition to $H(s)$ in Equation (1) and considering a source resistance $R_{S}$ and a capacitive load $C_{T}$, the Elmore delay for a distributed $R C$ or $R L C$ line model is

$$
T_{E D}=R_{S}\left(C+C_{T}\right)+R\left(\frac{C}{2}+C_{T}\right)
$$

By considering only one pole in the transfer function, i.e, approximating the denominator polynomial to only first moment, the single-pole response can be obtained as in [3]. The single pole of the transfer function is equal to the inverse of the Elmore delay $T_{E D}$. Hence, the delay at arbitrary thresholds of the single-pole response can be directly related to Elmore delay (Elmore delay corresponds to the $63.2 \%$ threshold delay of the single-pole response). 
For example, delay at $90 \%$ threshold voltage is $2.3 * T_{E D}$, i.e.,

$$
T_{0.9}=1.15 R C+2.3\left(R_{S}\left(C+C_{T}\right)+R C_{T}\right)
$$

Below, we use this expression to compute $90 \%$ threshold delay according to the Elmore model.

- Sakurai [14] also gives response and delay calculations for the distributed $R C$ line. He calculates the time-domain response from the transfer function using the Heaviside expansion over poles of the transfer function. Then, he approximates the response using a single pole and observes the variation of delay with respect to source and load parameters; a $90 \%$ threshold delay estimate is heuristically obtained as $T_{0.9}(h)=1.02 R C+2.3\left(R_{S}\left(C+C_{T}\right)+R C_{T}\right)$, quite similar to the Elmore delay equation (3).

Since these single pole delay estimates cannot accurately estimate delay for $R L C$ interconnects, Zhou et al. [17] proposed a two-pole approximation for the transfer function to compute the response at the load for $R L C$ interconnection trees. However, this technique does not provide any analytical expression for delay and is too time-consuming to be used in iterative layout optimization. Recently, [7] proposed to improve the Elmore delay model by using higher-order moments; this work gives a heuristic delay model equal to the sum of the first moment $\left(M_{1}\right)$ and its standard deviation $\left(\sqrt{\left|M_{1}^{2}-M_{2}\right|}\right){ }^{1}$

\section{A NEW ANALYTICAL DELAY MODEL}

We now develop a simple closed-form delay estimate, based on first and second moments, which to our knowledge is the first analytical delay model that handles arbitrary threshold voltages and inductance effects for a distributed line. We model an arbitrary interconnect line as follows: (i) the source is modeled as a resistive and inductive impedance $\left(Z_{S}=R_{S}+s L_{s}\right)$, and (ii) the load at the end of the interconnect line is modeled as a capacitive impedance $\left(Z_{T}=\frac{1}{s C_{T}}\right)$. Thus, the transfer function for the interconnect line of Figure 1 is given by Equation (1). We truncate this transfer function by expanding the hyperbolic functions around $s=0$; expansion around $s=\infty$ is not necessary since we consider only the first few coefficients of the transfer function. I.e., expanding cosh and $\sinh$ as infinite series and collecting terms up to the coefficient of $s^{2}$ in the denominator, we obtain the truncated transfer function

$$
H(s) \approx \frac{1}{1+s b_{1}+s^{2} b_{2}}
$$

with coefficients $b_{1}=R_{S} C+R_{I} C_{T}+\frac{R C}{2}+R C_{T}$ and $b_{2}=$ $\frac{R_{S} R C^{2}}{6}+\frac{R_{S} R C C_{T}}{2}+\frac{(R C)^{2}}{24}+\frac{R^{2} C C_{T}}{6}+L_{S} C+L_{S} C_{T}+\frac{L C}{2}+L C_{T}$ Note that the first and second moments of the transfer function can be obtained from the coefficients $b_{1}$ and $b_{2}$, i.e., $M_{1}=$ $b_{1}$ and $M_{2}=b_{1}^{2}-b_{2}$. (We use the coefficient notation $b_{1}, b_{2}$ and the moment notation $M_{1}, M_{2}$ interchangeably according to the simplicity of the expression. ) Depending on the sign of $b_{1}^{2}-4 b_{2}$, the poles of the transfer function can be either real or complex. We separately derive our delay model from the two-pole response for each of these cases.

\section{Real Poles:}

The two-pole methodology $[6,17]$ yields the following response for the case of real poles:

$$
v(t)=V_{0}\left(1-\frac{s_{2}}{s_{2}-s_{1}} e^{s_{1} t}+\frac{s_{1}}{s_{2}-s_{1}} e^{s_{2} t}\right)
$$

\footnotetext{
${ }^{1}$ In the early drafts of our paper [6] we also considered exactly the same model; however, we found that it is not as accurate as our

\begin{tabular}{|c|c|c|c|c|c|}
\hline$R_{S}$ & $L_{S}$ & $C_{T}$ & SPICE & Elmore & New Model \\
\hline$\Omega$ & $p H$ & $p F$ & $p s$ & $p s$ & $p s$ \\
\hline 50 & 246 & $0.776^{2}$ & 22.33 & 2293 & 22.21 \\
\hline 100 & 246 & 76 & 45.30 & 4520 & 15.70 \\
\hline 500 & 2,46 & 0.776 & 224.50 & 223.50 & 228.95 \\
\hline & & & 440.211 & & \\
\hline 25 & 246 & 76 & 107.10 & 708.40 & 708.65 \\
\hline 50 & 246 & .76 & 270.10 & 20.80 & \\
\hline 700 & 2,46 & .76 & 415,20 & 475,40 & 425.10 \\
\hline$\frac{500}{1000}$ & $\frac{240}{246}$ & $\frac{10}{66}$ & $\frac{20.32 .00}{409950}$ & $\frac{20.03 .00}{47000}$ & 40.30 \\
\hline
\end{tabular}
present model for various source and load parameters (see [6] for details).
}

Table 1. $90 \%$ threshold delay estimates for combinations of source and load parameters for which the poles of the response are real (i.e., overdamped response). The interconnect line parameters are $r=0.015 \Omega / \mu \mathrm{m}$, $l=0.246 \mathrm{pH} / \mu \mathrm{m}$ and $c=0.176 \mathrm{fF} / \mu \mathrm{m}$ and the length of the interconnect is $100 \mu \mathrm{m}$.

where $s_{1,2}=\frac{2}{-M_{1} \pm \sqrt{4 M_{2}-3 M_{1}^{2}}}$. The condition for the poles to be real is $\left(4 M_{2}-3 M_{1}^{2}\right) \geq 0$. Since $s_{2}-s_{1}=-\frac{\sqrt{4 M_{2}-3 M_{1}^{2}}}{M_{1}^{2}-M_{2}}$ is negative, the coefficients $\frac{s_{2}}{s_{2}-s_{1}}$ and $\frac{s_{1}}{s_{2}-s_{1}}$ are positive. Also, since the magnitude $\left|s_{2}\right|$ is greater than $\left|s_{1}\right|$, the second term in the response decreases rapidly compared to the first term. Hence, the two-pole response can be lower-bounded as

$$
v(t) \approx V_{0}\left(1-\frac{s_{2}}{s_{2}-s_{1}} e^{s_{1} t}\right)
$$

Since the voltage is lower-bounded, the delay obtained is an upper bound on the actual delay. The delay $\tau_{r}$ (the subscript indicates the case of real poles) at threshold voltage $v_{t h}$ is

$$
\tau_{r}=\frac{K_{r}}{\left|s_{1}\right|}=K_{r} \frac{M_{1}+\sqrt{4 M_{2}-3 M_{1}^{2}}}{2}
$$

where $K_{r}$ is a function of the coefficients $b_{1}$ and $b_{2}$, i.e., $K_{r}=\ln \left(\frac{1}{2\left(1-v_{t h}\right)}\left[1+\frac{b_{1}}{\sqrt{b_{1}^{2}-4 b_{2}}}\right]\right)$. For the wide range of source, load and interconnect parameter values considered in our simulations (see Table 1), we find that $K_{r}$ for $v_{t h}=0.90$ is actually almost a constant, i.e., $K_{r}=2.36$ gives a very strong fit between SPICE delay values ${ }^{2}$ and $\frac{1}{\left|s_{1}\right|}[6]$. Thus, we use

$$
\tau_{r}=2.36 * \frac{\left(M_{1}+\sqrt{4 M_{2}-3 M_{1}^{2}}\right)}{2} ;
$$

the resulting delay estimates are compared against those of various other methods in Table 1. Our analytical delay model gives estimates close to those obtained from SPICE, but Elmore delay also gives good estimates for this case of overdamped response.

\section{Complex Poles}

The condition for complex poles is $\left(4 M_{2}-3 M_{1}^{2}\right)=\left(b_{1}^{2}-\right.$ $\left.4 b_{2}\right) \leq 0$. The time-domain response for complex poles [5] is

$$
v(t)=V_{0}\left(1-\frac{\sqrt{\alpha^{2}+\beta^{2}}}{\beta} e^{-\alpha t} * \sin (\beta t+\rho)\right)
$$

where $\alpha=\frac{M_{1}}{2\left(M_{1}^{2}-M_{2}\right)}, \beta=\frac{\sqrt{3 M_{1}^{2}-4 M_{2}}}{2\left(M_{1}^{2}-M_{2}\right)}$ and $\rho=\tan ^{-1}\left(\frac{\beta}{\alpha}\right)$. Using the above equation and threshold voltage $v_{t h}$, we get

$$
e^{-\alpha t} * \sin (\beta * t+\rho)=\frac{1-v_{t h}}{\sqrt{1+\left(\frac{\alpha}{\beta}\right)^{2}}} .
$$

${ }^{2}$ SPICE simulation results are obtained using SPICE3 and the built-in LTRA (lossy transmission line) model, which is based on convolution techniques [12]. 


\begin{tabular}{||c|c|c|c|c|c||}
\hline \hline$R_{S}$ & $L_{S}$ & $C_{T}$ & SPTCE & EImore & New ModeI \\
\hline$\Omega$ & $p H$ & $p F$ & $p s$ & $p s$ & $p s$ \\
\hline \hline 10 & 0.0246 & 0.0176 & 1.22 & 0.90 & 1.30 \\
\hline 15 & 0.0246 & 0.0176 & 1.33 & 1.31 & 1.38 \\
\hline 20 & 0.0246 & 0.0176 & 1.47 & 1.71 & 1.51 \\
\hline 25 & 0.0246 & 0.0176 & 1.60 & 2.12 & 1.64 \\
\hline \hline 10 & 0.0246 & 0.176 & 4.50 & 5.12 & 4.25 \\
\hline 15 & 0.0246 & 0.176 & 5.85 & 7.32 & 5.31 \\
\hline 20 & 0.0246 & 0.176 & 7.90 & 9.55 & 8.60 \\
\hline \hline 10 & 2.46 & 0.0176 & 1.31 & 0.90 & 1.40 \\
\hline 15 & 2.46 & 0.0176 & 1.40 & 1.31 & 1.49 \\
\hline 20 & 2.46 & 0.0176 & 1.55 & 1.71 & 1.59 \\
\hline 25 & 2.46 & 0.0176 & 1.63 & 2.12 & 1.69 \\
\hline \hline 10 & 2.46 & 0.176 & 4.65 & 5.10 & 4.30 \\
\hline 15 & 2.46 & 0.176 & 5.85 & 7.33 & 5.30 \\
\hline 20 & 2.46 & 0.176 & 7.98 & 9.55 & 8.70 \\
\hline \hline 10 & 24.6 & 0.0176 & 1.80 & 0.90 & 1.96 \\
\hline 15 & 24.6 & 0.0176 & 1.89 & 1.31 & 2.06 \\
\hline 20 & 24.6 & 0.0176 & 2.00 & 1.71 & 2.15 \\
\hline 25 & 24.6 & 0.0176 & 2.19 & 2.11 & 2.21 \\
\hline \hline 10 & 24.6 & 0.1776 & 5.65 & 5.10 & 5.44 \\
\hline 15 & 24.6 & 0.176 & 6.50 & 7.33 & 5.95 \\
\hline 20 & 24.6 & 0.176 & 7.66 & 9.55 & 6.97 \\
\hline 25 & 24.6 & 0.176 & 9.47 & 11.78 & 9.26 \\
\hline \hline & & & & & \\
\hline
\end{tabular}

Table 2. $90 \%$ threshold delay estimates for combinations of source and load parameters for which the poles of the response are complex (i.e., underdamped response). The interconnect line parameters are $r=0.015$ $\Omega / \mu \mathrm{m}, l=0.246 \mathrm{pH} / \mu \mathrm{m}$ and $c=0.176 \mathrm{fF} / \mu \mathrm{m}$ and the length of the interconnect is $100 \mu \mathrm{m}$.

The delay at a given threshold voltage can be computed by solving for time in Equation (5) recursively. One way to solve the recursive Equation (5) is to approximate the time variable in the exponential term by Elmore delay, i.e., substitute $T_{E D}$ for time $t$. Expanding sine as a Taylor series and considering only the first term yields

$$
\tau_{c}=\frac{K_{c}}{\beta}=K_{c} * \frac{2\left(M_{1}^{2}-M_{2}\right)}{\sqrt{3 M_{1}^{2}-4 M_{2}}}
$$

where $K_{c}=\frac{\left(1-v_{t h}\right) e^{\alpha * T_{E D}}}{\sqrt{1+\left(\frac{\alpha}{\beta}\right)^{2}}}-\rho$. Even though $K_{c}$ is a function of $b_{1}$ and $b_{2}$, for a wide range of interconnect, source and load parameters it too is almost a constant, i.e., $K_{c}=1.66$ gives a good fit between SPICE delay values and $\frac{1}{\beta}[6]$. In other words, our $90 \%$ threshold delay estimate for complex poles is

$$
\tau_{c}=1.66 \cdot \frac{2\left(M_{1}^{2}-M_{2}\right.}{\sqrt{3 M_{1}^{2}-4 M_{2}}} .
$$

Table 2 shows delay values for various combinations of source, load and interconnect parameters assuming this value of $K_{c}$ The delay estimates using our analytical model are within $15 \%$ of SPICE-computed delay estimates, while Elmore delay estimates vary by as much as $33 \%$ from SPICE-computed delays. Hence, for the case of complex poles (i.e., underdamped response), the Elmore model is no longer acceptably accurate.

As detailed in [6], we have also considered the special case in which poles are equal, i.e., a double pole configuration. The delay at $90 \%$ threshold for this case can be obtained as $\tau_{0.9}=K_{d} \frac{b_{1}}{2}$, which gives a recursive equation for $K_{d}$, i.e., $K_{d}=\ln \left(10\left(1+K_{d}\right)\right)$ from which $K_{d} \approx 3.9$. Thus, in the case of a double pole we estimate the $90 \%$ threshold delay as

$$
\tau_{0.9}=K_{d} \cdot \frac{b_{1}}{2}=1.95 b_{1}
$$

which is independent of the inductance value and different from the Elmore delay expression.

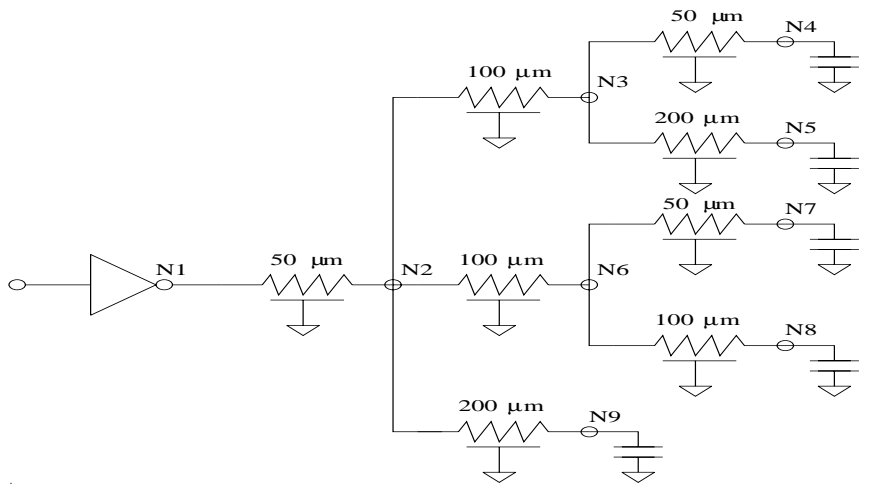

Figure 2. A simple interconnection tree consisting of distributed $R L C$ lines.

\section{INTERCONNECTION TREES}

We conclude with an extension of our analytical model to estimate delays in arbitrary interconnect trees. An $R L C$ network is called an $R L C$ tree if it does not contain a closed path of resistors and inductors, i.e., all resistors and inductors are floating with respect to ground and all capacitors are connected to ground. Consider an $R L C$ interconnect tree with root (or source) $S$ and set of sinks (or leafs) $L=\left\{L_{1}, L_{2}, \ldots, L_{n}\right\}$. The unique path from root $S$ to sink node $i$ is denoted by $p(i)$ and is referred to as the main path. The edges/nodes not on the main path are referred as the off-path edges/nodes. We model each edge on the main path of the tree using a lumped $R L C$ segment, e.g., an $\mathbf{L}, \mathbf{T}$, or $\Pi$ model. We replace the off-path subtree rooted at node $j$ with the total subtree capacitance at node $j$. (Figure 3 shows an example of a main path where each branch in the tree is replaced by $R L C$ segments, and the off-path subtrees are replaced by their respective subtree capacitances.) At any node $j$, the total capacitance is given by

$C^{\prime}=C_{j} \quad$ if no off-path subtree at node $j$

$C_{j}^{\prime}=C_{j}+C_{T(j)} \quad$ if node $K$ has off-path subtree $T(j)$

where $C_{j}$ is the capacitance at the node and $C_{T(j)}$ is the offpath subtree capacitance at node $j$. The $k^{\text {th }}$ coefficient $b_{k}$ of the transfer function for the general $R L C$ circuit of Figure 3 can be expressed using the following recursive equation [5]:

$$
b_{k}^{M}=R_{M-1} \sum_{j=1}^{M-1} C_{j}^{\prime} \cdot b_{k-1}^{j}+L_{M-1} \sum_{j=1}^{M-1} C_{j}^{\prime} \cdot b_{k-2}^{j}+b_{k}^{M-1}
$$

where $b_{K}^{M}$ refers to the coefficient of $s^{k}$ in the transfer function

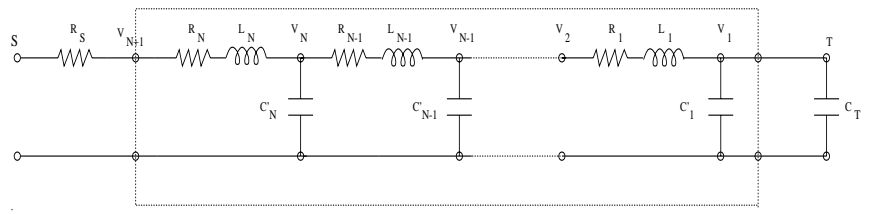

Figure 3. Representation of the main path in the tree, where each distributed line is modeled using $R L C$ segments.

between node $M$ and node 1 . Note that $b_{0}^{j}=1, b_{-1}^{j}=0$ for all $j$ and $b_{k}^{1}=0$ for all $k$. Using the above recursive equation the expressions for the first and second coefficients of the transfer function at the root can be derived as

$$
b_{1}^{N+1}=R_{N} \sum_{j=1}^{N} C_{j}^{\prime}+b_{1}^{N}=\sum_{i=1}^{N} R_{i} \sum_{j=1}^{i} C_{j}^{\prime}
$$




$$
b_{2}^{N+1}=\sum_{j=2}^{N} C_{j}^{\prime} \sum_{l=j}^{N} R_{l} \sum_{i=1}^{j-1} C_{j}^{\prime} \sum_{d=i}^{j-1} R_{d}+\sum_{j=1}^{N} C_{j}^{\prime} \sum_{l=j}^{N} L_{l}
$$

For any given source and sink pair the coefficients $b_{1}$ and $b_{2}$ can be computed in linear time by traversing the main path and using the above recursive equation. Using the analytical delay model developed in the previous section, we can obtain an analytical delay estimate for $R L C$ interconnect trees using the first and second coefficients. Thus, the $90 \%$ threshold delay at a given sink $i$, depending on the value of $\left(4 M_{2}-3 M_{1}^{2}\right)$, is

$$
\begin{aligned}
& =K_{r} \cdot \frac{\left(M_{1}+\sqrt{4 M_{2}-3 M_{1}^{2}}\right)}{2} & & \text { for Real poles } \\
T_{N D}(i) & =K_{c} \cdot \frac{2\left(M_{1}^{2}-M_{2}\right)}{\sqrt{3 M_{1}^{2}-4 M_{2}}} & & \text { for Complex poles } \\
& =K_{d} \cdot \frac{M_{1}}{2} & & \text { for Double poles }
\end{aligned}
$$

where the first and second moments are expressed as $M_{1}=b_{1}$ and $M_{2}=b_{1}^{2}-b_{2}$. The coefficients of the transfer function are obtained from Equation (9). By contrast, 90\% threshold delay according to the Elmore model is simply $T_{E D}(i)=2.3 * M_{1}$.

We evaluate our analytical model by considering the simple interconnection tree shown in Figure 2. We consider the sink node $N 4$ for delay estimation. Each edge on the main path between the root and node $N 4$ is replaced by a two- $\mathbf{L}$ segment model. ${ }^{3}$ We then apply the above recursive coefficient computation for the resultant $R L C$ circuit of the main path. The $90 \%$ threshold delays according to both the Elmore model and our new analytical model (Equation (10)) are then computed. We also compute the delay at the given sink node using SPICE3e, where each edge of the tree is modeled using the LTRA (Lossy Transmission Line) model (with SPICE, we first compute the response at the sink node and then find the delay for $90 \%$ threshold voltage). Table 3 compares delay estimates over a range of interconnect parameters, driver resistance values, and sink load capacitance values: Elmore delay varies by as much as $35 \%$ from the SPICE-computed delay, but our new model is within $18 \%$ of the SPICE delay for all examples. Note that our delay estimates also require three orders of magnitude less computation than SPICE, since they have the same time complexity as the Elmore delay estimate.

\section{CONCLUSIONS}

Fast delay estimation methods, as opposed to simulation techniques, are needed for incremental performance-driven layout synthesis. Elmore delay based estimation methods, although efficient, cannot accurately estimate the delay for $R L C$ interconnect lines. We have obtained an analytical delay model, based on first and second moments of $R L C$ interconnection lines, which considers the effect of inductance. The resulting delay estimates are significantly more accurate than Elmore delay estimates We also extend our delay model to estimate source-sink delays in arbitrary interconnect trees. For the small tree topology considered, we observe improvement of at least $18 \%$ in the accuracy of our delay estimates, compared to the Elmore model. Since our model has the same time complexity as the Elmore model, we believe it can be valuable in modern iterative layout synthesis methodologies. Ongoing work applies our analytical model to delay-driven routing tree construction, zero-skew routing, and delay estimation in nets spanning multiple routing layers (i.e., with modeling of vias).

\section{REFERENCES}

[1] L. N. Dworsky, Modern Transmission Line Theory and Applications, Wiley, 1979.

${ }^{3}$ Our model is not limited to traditional segment models, and indeed we believe the accuracy of our results would improve if we use non-uniform segment models [5] designed to perfectly match the low-order moments of the distributed $R L C$ line.

\begin{tabular}{||c|c|c|c|c||}
\hline \hline $\begin{array}{c}\text { Line Para. } \\
(\ell \mu \mathrm{m})\end{array}$ & $\begin{array}{c}C_{L} \\
(p F)\end{array}$ & \multicolumn{3}{|c||}{ Delay $(p s)$} \\
\cline { 3 - 5 } & SPICE & Elmore & New Model \\
\hline \hline $\begin{array}{c}R=0.015 \Omega \\
C=0.176 f F \\
L=0.246 p H\end{array}$ & 0.02 & 5.7 & 6.6 & 5.0 \\
\hline $\begin{array}{c}R=0.0015 \Omega \\
C=0.176 f F \\
L=2.46 p H\end{array}$ & 0.2 & 37 & 26 & 31 \\
\hline $\begin{array}{c}R=0.015 \Omega \\
C=0.176 f F \\
L=2.46 p H\end{array}$ & 0.2 & 39 & 29 & 32 \\
\hline $\begin{array}{c}R=0.0075 \Omega \\
C=0.176 f F \\
L=2.46 p H\end{array}$ & 2.0 & 179 & 238 & 205 \\
\hline $\begin{array}{c}R=0.0015 \Omega \\
C=0.176 f F \\
L=0.246 p H\end{array}$ & 2.0 & 231 & 238 & 232 \\
\hline $\begin{array}{c}R=0.015 \Omega \\
C=0.176 f F \\
L=2.46 p H\end{array}$ & 2.0 & 199 & 270 & 230 \\
\hline \hline
\end{tabular}

Table 3. $90 \%$ threshold delay values for a wide range of interconnect parameters at Node 4 of the tree in Figure 5. The driver resistance is $R_{S}=10 \Omega$. We compare SPICE LTRA, and the Elmore model, against our analytical delay model.

[2] W. C. Elmore, "The Transient Response of Damped Linear Networks with Particular Regard to Wideband Amplifiers", Journal of Applied Physics 19, Jan. 1948, pp. 55-63.

[3] M. A. Horowitz, "Timing Models for MOS Circuits", PhD Thesis, Stanford University, Jan. 1984.

[4] C. C. Huang and L. L. Wu, "Signal Degradation Through Module Pins in VLSI Packaging", IBM J. Res. and Dev. 31(4), July 1987, pp. 489-498.

[5] A. B. Kahng and S. Muddu, "Two-pole Analysis of Interconnection Trees", Proc. IEEE MCMC Conf., January 1995, pp. 105-110.

[6] A. B. Kahng and S. Muddu, "Accurate Analytical Delay Models for VLSI Interconnections", UCLA CS Dept. TR-950034, Sep. 1995 (see also TR-940015).

[7] B. Krauter, R. Gupta, J. Willis, and L. T. Pileggi, "Transmission Line Synthesis", Proc. 32th ACM/IEEE Design Automation Conf., June 1995, pp. 358-363.

[8] S. Lin and E. S. Kuh, "Transient Simulation of Lossy Interconnect", Proc. 29th ACM/IEEE Design Automation Conf., June 1992, pp. 81-86.

[9] S. P. McCormick and J. Allen, "Waveform Moment Methods for Improved Interconnection Analysis", Proc. 27th ACM/IEEE Design Automation Conf., June 1990, pp. 406-412.

[10] L. T. Pillage and R. A. Rohrer, "Asymptotic Waveform Evaluation for Timing Analysis", IEEE Trans. on CAD 9, Apr. 1990, pp.352-366.

[11] C. L. Ratzlaff, N. Gopal, and L. T. Pillage, "RICE: Rapid Interconnect Circuit Evaluator", Proc. 28th ACM/IEEE Design Automation Conf., June 1991, pp. 555-560.

[12] J. S. Roychowdhury and D. O. Pederson, "Efficient Transient Simulation of Lossy Interconnect", Proc. 28th ACM/IEEE Design Automation Conf., June 1991, pp. 740-745.

[13] J. Rubinstein, P. Penfield and M. A. Horowitz, "Signal Delay in RC Tree Networks", IEEE Trans. on CAD 2(3), July 1983, pp. 202-211.

[14] T. Sakurai, "Approximation of Wiring Delay in MOSFET LSI", IEEE Journal of Solid-State Circuits, Aug. 1983, Vol.18, No.4, pp. $418-426$.

[15] T. Sakurai, "Closed-Form Expressions for Interconnection Delay, Coupling, and Crosstalk in VLSI's", IEEE Trans. on Electron Devices 40, Jan. 1993, pp. 118-124.

[16] M. Sriram and S. M. Kang, "Fast Approximation of The Transient Response of Lossy Transmission Line Trees", Proc. ACM/IEEE Design Automation Conf., June 1993, pp. 691-696.

[17] D. Zhou, S. Su, F. Tsui, D. S. Gao and J. S. Cong, "A Simplified Synthesis of Transmission Lines with A Tree Structure", Intl. Journal of Analog Integrated Circuits and Signal Processing 5, Jan. 1994, pp. 19-30. 